OPEN ACCESS

Edited by:

Ivan Argatov,

Technische Universität Berlin,

Germany

Reviewed by:

Sergei Bosiakov,

Belarusian State University, Belarus

Gennaro Vitucci,

Catholic University of Louvain,

Belgium

*Correspondence:

Cynthia J. Roberts

roberts.8@osu.edu

Specialty section: This article was submitted to Biomechanics,

a section of the journal Frontiers in Bioengineering and Biotechnology

Received: 12 October 2018 Accepted: 18 December 2018 Published: 10 January 2019

Citation:

Nguyen BA, Roberts CJ and Reilly MA (2019) Biomechanical Impact of the Sclera on Corneal Deformation Response to an Air-Puff: A Finite-Element Study.

Front. Bioeng. Biotechnol. 6:210. doi: 10.3389/fbioe.2018.00210

\section{Biomechanical Impact of the Sclera on Corneal Deformation Response to an Air-Puff: A Finite-Element Study}

\author{
B. Audrey Nguyen ${ }^{1}$, Cynthia J. Roberts ${ }^{1,2 *}$ and Matthew A. Reilly ${ }^{1,2}$ \\ ${ }^{1}$ Department of Biomedical Engineering, The Ohio State University, Columbus, OH, United States, ${ }^{2}$ Department of \\ Ophthalmology \& Visual Science, The Ohio State University, Columbus, OH, United States
}

Aim or Purpose: To describe the effect of varying scleral stiffness on the biomechanical deformation response of the cornea under air-puff loading via a finite-element (FE) model.

Methods: A two-dimensional axisymmetric stationary FE model of the whole human eye was used to examine the effects varying scleral stiffness and intraocular pressure (IOP) on the maximum apical displacement of the cornea. The model was comprised of the cornea, sclera, vitreous, and surrounding air region. The velocity and pressure profiles of an air-puff from a dynamic Scheimpflug analyzer were replicated in the FE model, and the resultant profile was applied to deform the cornea in a multiphysics study (where the air-puff was first simulated before being applied to the corneal surface). IOP was simulated as a uniform pressure on the globe interior. The simulation results were compared to data from ex vivo scleral stiffening experiments with human donor globes.

Results: The FE model predicted decreased maximum apical displacement with increased IOP and increased ratio of scleral-to-corneal Young's moduli. These predictions were in good agreement (within one standard deviation) with findings from ex vivo scleral stiffening experiments using human donor eyes. These findings demonstrate the importance of scleral material properties on the biomechanical deformation response of the cornea in air-puff induced deformation.

Conclusion: The results of an air-puff induced deformation are often considered to be solely due to IOP and corneal properties. The current study showed that the stiffer the sclera, the greater will be the limitation on corneal deformation, separately from IOP. This may have important clinical implications to interpreting the response of the cornea under air-puff loading in pathologic conditions.

Keywords: sclera, biomechanics, air-puff, finite-element, deformation

\section{INTRODUCTION}

Biomechanical markers are being explored to improve screening, diagnosis, and management of diseases such as keratoconus and glaucoma (Liu and Roberts, 2005; Elsheikh et al., 2009; Ruberti et al., 2011; Coudrillier et al., 2012; Tang and Liu, 2012; Hon and Lam, 2013; Metzler et al., 2014; Girard et al., 2015; Sinha Roy et al., 2015; Ariza-Gracia et al., 2016; Roberts, 2016). Biomechanical properties of the sclera are often determined via ex vivo mechanical strip testing or inflation tests 
(Coudrillier et al., 2012; Geraghty et al., 2012; Girard et al., 2015; Pandolfi and Boschetti, 2015; Nguyen, 2016). However, this destructive method depends on a multitude of factors including tissue hydration and strain rate, and there is a wide range of reported values in the literature on the value of Young's modulus of the sclera (Coudrillier et al., 2012). There is a demonstrated need for a clinical tool to determine the in vivo biomechanical behavior of ocular tissues, including how the cornea and the sclera interact.

It has been shown that the biomechanical response of the cornea under air-puff deformation is significantly affected by its boundary properties (Elsheikh, 2010; Metzler et al., 2014). Metzler et al. showed that the human cornea behaves more stiffly in the case of a corneoscleral button mounted on a rigid artificial anterior chamber (simulating stiffer scleral material properties) as opposed to a cornea that remains connected to an intact whole globe at physiologically normal values of IOP. Their results suggest that the scleral properties have an important impact on the deformation response of the cornea under air-puff loading (Metzler et al., 2014).
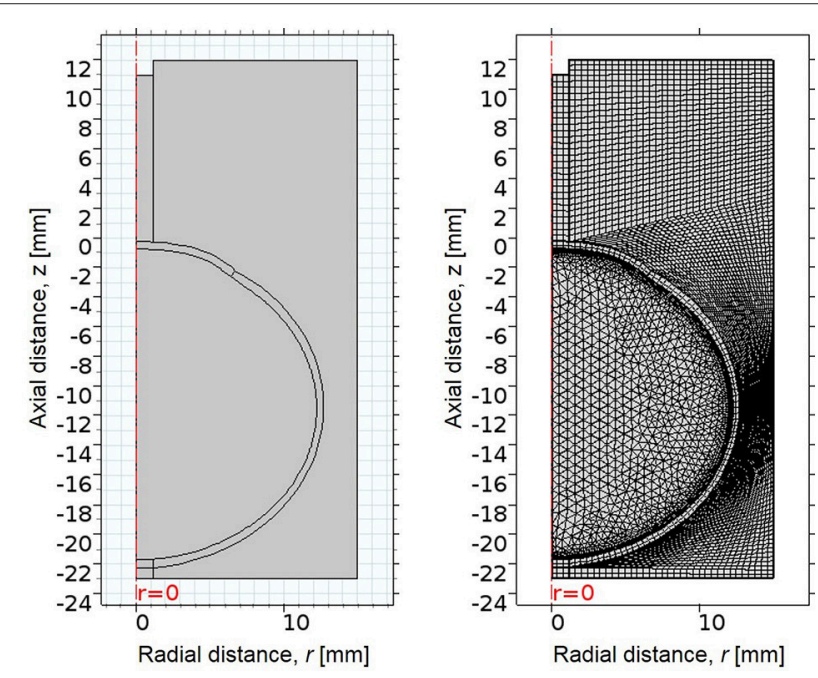

FIGURE 1 | (Left) 2D axisymmetric geometry of eye, consisting of cornea, sclera, and vitreous, surrounded by an air region. The $\mathrm{x}$-axis indicates radial distance from the line of rotational symmetry, and the $y$-axis indicates axial distance, where the undeformed corneal apex is at $0 \mathrm{~mm}$. (Right) The mesh of the FE model, consisting of quadrilateral and free triangular elements.

TABLE 1 | Summary of geometric parameters for finite element model.

\begin{tabular}{lll}
\hline Tissue & Geometric parameter & Parameter value \\
\hline Cornea & Central corneal thickness & $500 \mu \mathrm{m}$ (Elsheikh and Wang, 2007) \\
& Anterior radius of curvature & $8.0 \mathrm{~mm}$ (Vojniković et al., 2013) \\
& Posterior radius of curvature & $6.8 \mathrm{~mm}$ (Vojniković et al., 2013) \\
Sclera & Thickness at equator & $400 \mu \mathrm{m}$ (Norman et al., 2010) \\
& Thickness at posterior pole & $1,000 \mu \mathrm{m}$ (Norman et al., 2010) \\
& Radius of curvature & $12 \mathrm{~mm}$ (Norman et al., 2010)
\end{tabular}

Two commercial devices have been developed to assess the biomechanical deformation response of the cornea using an airpuff as the non-destructive load, and they have been used to provide clinical insight into the in vivo biomechanical behavior in pathological conditions (Luce, 2005; Ambrósio et al., 2013). The data produced by these devices are often interpreted as purely corneal response without considering the contribution of the sclera. Therefore, our purpose is to develop a whole-eye finiteelement model that utilizes a well-characterized air-puff as the load to investigate the impact of varying scleral properties on corneal deformation response. This model will be validated using ex vivo experimental data obtained using human donor eyes.

\section{METHODS}

The CorVis ST (Oculus Optikgeräte, GmbH, Wetzlar, Germany) utilizes a high-speed camera with Scheimpflug geometry at a frame rate of $4,300 \mathrm{~Hz}$ to acquire 140 images of the cornea along a single horizontal meridian, providing dynamic corneal deformation response parameters, and visualization of the cornea as it deforms (Roberts, 2014). Additionally, the air-puff of the CorVis ST has been shown to be consistent and repeatable (Roberts et al., 2017), and for these reasons was chosen as the load for the FE model. The ability to apply an accurate and precise load while monitoring deformation is the hallmark of mechanical testing.

\section{Geometry and Mesh}

A simplified axisymmetric FE model of the human eye was developed in COMSOL Multiphysics 5.2a (COMSOL Inc.; Burlington, MA) based on average dimensions (Figure 1, Table 1). The whole-eye geometry consisted of cornea, sclera, and vitreous humor. The whole globe has a diameter of $24 \mathrm{~mm}$, the cornea has a diameter of $11 \mathrm{~mm}$, and the central corneal thickness

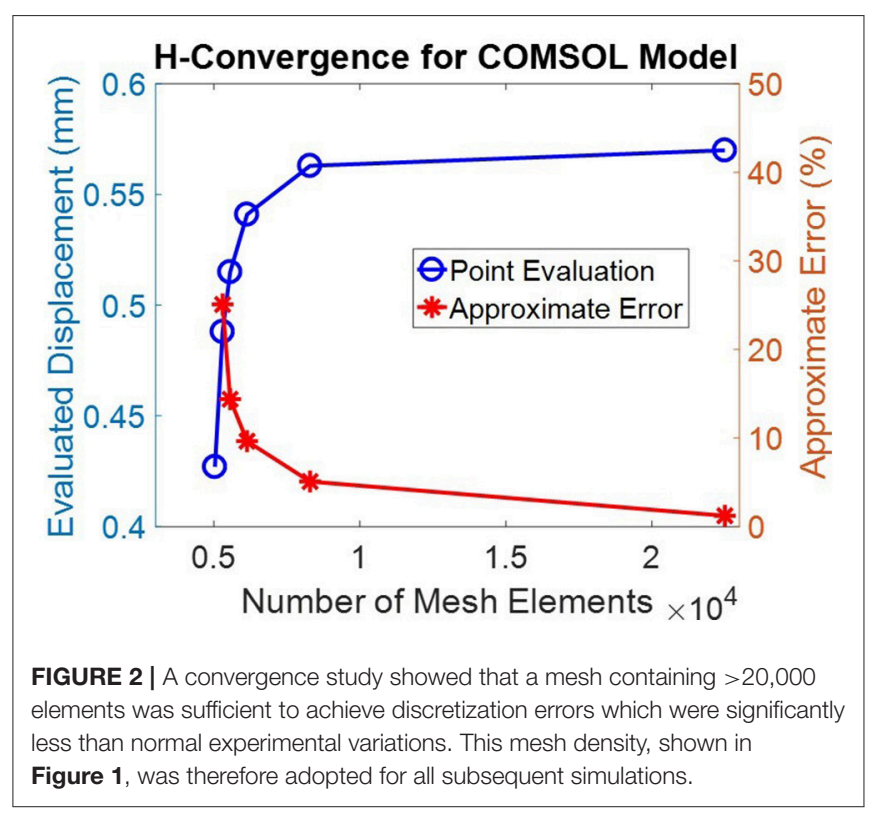


(CCT) is $500 \mu \mathrm{m}$. The thickness of the sclera ranged from 400 to $1,000 \mu \mathrm{m}$.

The geometry was meshed using the built-in meshing capabilities of COMSOL. The central air region and ocular tunic were meshed with mapped 12,837 quadrilateral elements, with 3 elements through the thickness of the cornea and sclera. The remaining air region domains were meshed with 7,453 free triangular elements. The globe interior was meshed using free triangular elements and boundary layers to create a highly refined mesh at the interface of the ocular tunic and the vitreous body and more accurately model corneal deformation response. A convergence study was undertaken to identify the mesh density required to ensure that the solution was nearly independent of meshing parameters (Figure 2).

\section{Estimation of Stress-Free Geometry}

The eye is naturally under tension due to loading by intraocular pressure (IOP); therefore the unloaded or stress-free state of the eye was first estimated (Elsheikh et al., 2013). A negative pressure equal to the IOP was applied to the interior boundaries of the ocular tunic to generate the unloaded state. The resultant stress-free geometry was then loaded by IOP to determine the residually-stressed state of the eye (Figure 3). This residuallystressed geometry was used as the starting point for the multiphysics FE simulation.

\section{Material Properties and Boundary Conditions}

The multiphysics model included the air-puff from the CorVis ST, which acted as the anterior load on the eye. The air-puff velocity and pressure profiles acting on the anterior cornea were simulated using turbulent $\mathrm{k}-\omega$ shear stress transport fluid flow physics. The maximum velocity at the nozzle outlet was set to $140.2 \mathrm{~m} / \mathrm{s}$ based on data from the characterization performed by Roberts et al. (2017). The remaining edges of the air region were set to be open boundaries, such that the air flow is not affected by the arbitrary region boundaries. The resultant pressure profile from the air-puff simulation was applied as the load on the anterior cornea to simulate corneal deformation. The central $1.5 \mathrm{~mm}$ radius of the posterior sclera was held fixed.

The material properties of the ocular tunic were described by an isotropic, nearly incompressible, neo-Hookean constitutive
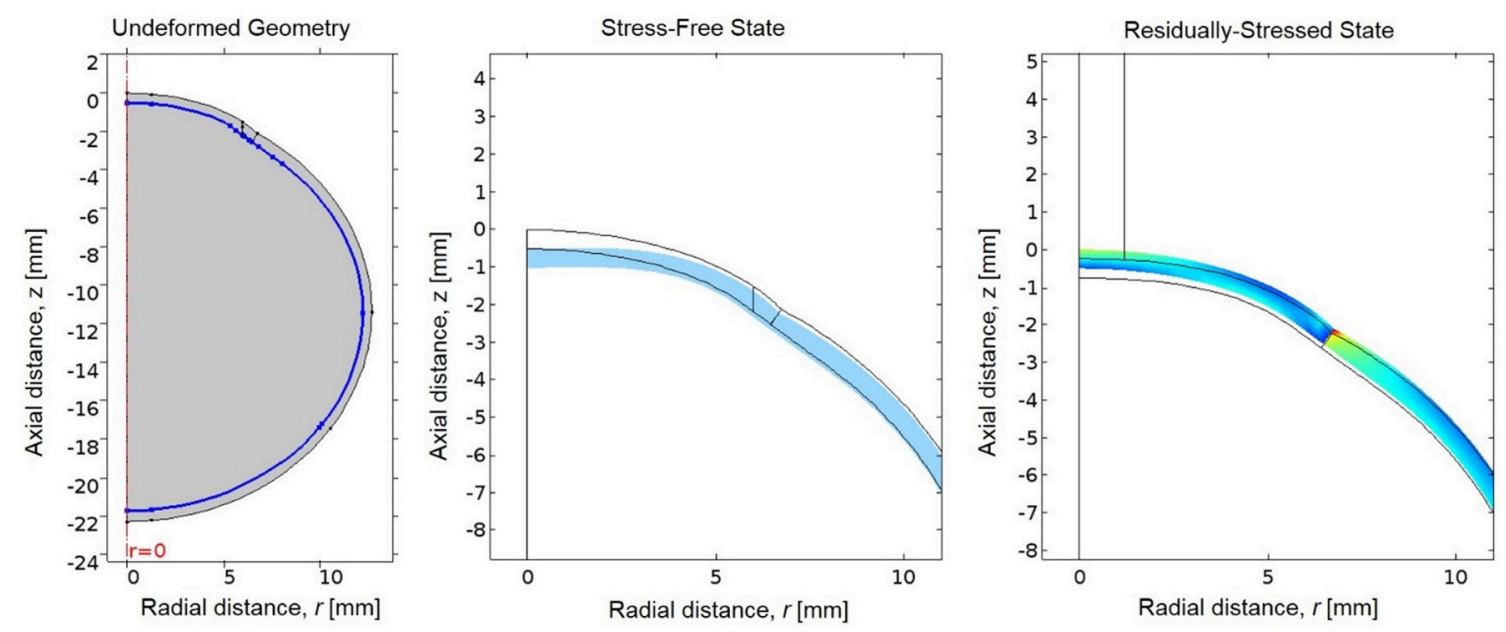

FIGURE 3 | (Left) The undeformed geometry of the eye, where the interior boundary of the ocular tunic is highlighted in blue to indicate where the negative IOP will be applied to estimate the stress-free geometry of the eye. (Center) The resultant stress-free state of the eye (in blue) compared to the original undeformed geometry prior to loading by the negative IOP (in black). (Right) Von Mises stresses in the residually-stressed state of the eye when loaded by IOP, where the black lines represent the geometry of the stress-free state of the eye prior to loading by IOP.

TABLE 2 | Material properties of ocular tissues in the FE model.

\begin{tabular}{llll}
\hline & Cornea & Sclera & Vitreous \\
\hline Young's modulus [MPa] & $\begin{array}{l}1.5 \text { (Hamilton and Pye, } \\
\text { 2008; McKee et al., }\end{array}$ & $\begin{array}{l}\text { 2.25, 3.0, 4.5, 6.0 } \\
\text { (McKee et al., 2011) }\end{array}$ & - \\
2011) & 0.49 & - \\
Poisson ratio & 0.49 & 76.01 & 0.49 \\
Bulk modulus [MPa] & 50.68 & & 0.375 (Heys et al., \\
Shear modulus $[\mathrm{Pa}]$ & - & - & $2001)$ \\
Density $\left[\mathrm{kg} / \mathrm{m}^{3}\right]$ & 1,050 (Ng and Ooi, & $1,100(\mathrm{Ng}$ and Ooi, & 0.75 (Heys et al., 2001) \\
Viscosity $\left[\mathrm{Pa}{ }^{*} \mathrm{~s}\right]$ & $2006)$ & $2006)$ & 1,000 (Heys et al.,
\end{tabular}


model with values estimated from the literature (Heys et al., 2001; $\mathrm{Ng}$ and Ooi, 2006; McKee et al., 2011). The vitreous was modeled as a linear elastic solid (Heys et al., 2001). Table 2 summarizes the material properties utilized in the FE model. The air-puff region surrounded the eye, which was fixed at the back to replicate the mounted whole-globe setup used in the ex vivo validation studies. The Young's modulus of the cornea was fixed at a representative value from the literature (Hamilton and Pye, 2008; McKee et al., 2011) while the Young's modulus of the sclera was varied to examine its relative effect on corneal deformation (Heys et al.,

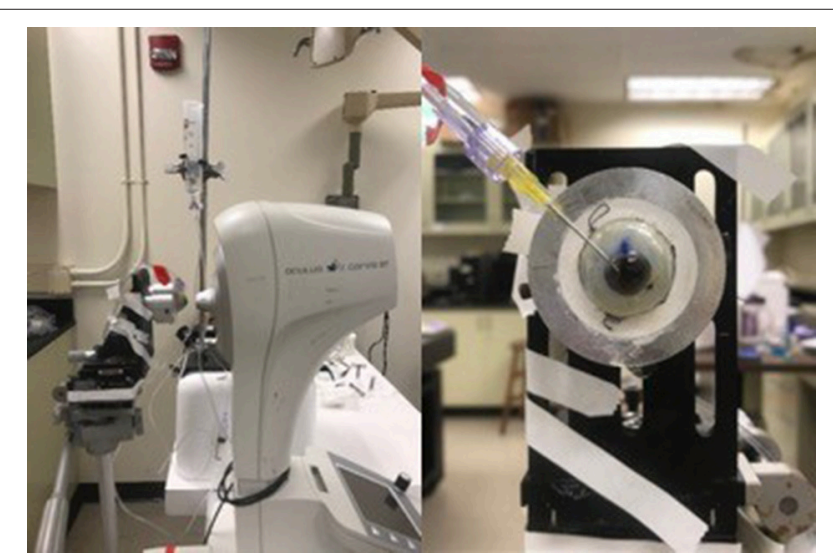

FIGURE 4 | (Left) Experimental setup for ex vivo studies on human donor eyes, showing a whole globe in the purpose-designed mount in front of the CorVis ST. (Right) A view of the human donor eye showing the 22-gauage needle inserted into the anterior chamber, used to set and maintain intraocular pressure.
2001; Ng and Ooi, 2006). Intraocular pressure (IOP) was varied from 10 to $40 \mathrm{mmHg}$ in $10 \mathrm{mmHg}$ increments. The properties of air, cornea, and vitreous were kept constant between simulations.

In the ex vivo setup of human donor studies, which was replicated by the finite element model, the peak of corneal motion/deformation is in phase with the peak of the air-puff loading curve. As the loading rate $(\mathrm{dP} / \mathrm{dt})$ approaches 0 , this aligns with the maximum apical displacement as measured by the CorVis ST. We therefore assumed, as a first approximation, the cornea is in a quasi-static equilibrium such that a static model will capture this particular aspect of the air-puff-induced deformation.

\section{Ex vivo Experiments}

The CorVis ST was used to acquire data on the biomechanical deformation response of the corneas of 12 pairs of human donor eyes ( $65 \pm 11.4$ years, 8 male/ 4 female). The donor eye study is considered exempt from review by the university. In each pair, one eye was randomly selected to have its sclera stiffened by crosslinking treatment with glutaraldehyde, while the fellow eye served as a control. The globe was immersed for $30 \mathrm{~min}$ in $4 \%$

TABLE 3 | Spatial measurements of air flow velocity at $4 \mathrm{~mm}$ from nozzle at peak pressure.

\begin{tabular}{lccc}
\hline $\begin{array}{l}\text { Radial distance, } \\
\mathbf{r}[\mathbf{m m}]\end{array}$ & $\begin{array}{c}\text { Simulation } \\
{[\mathrm{m} / \mathrm{s}]}\end{array}$ & $\begin{array}{c}\text { Hot wire (Roberts } \\
\text { et al., 2017) [m/s] }\end{array}$ & $\begin{array}{c}\text { Error } \\
{[\%]}\end{array}$ \\
\hline 0.75 & 120.496 & 120.734 & -0.196 \\
1.50 & 50.601 & 50.594 & 0.015
\end{tabular}
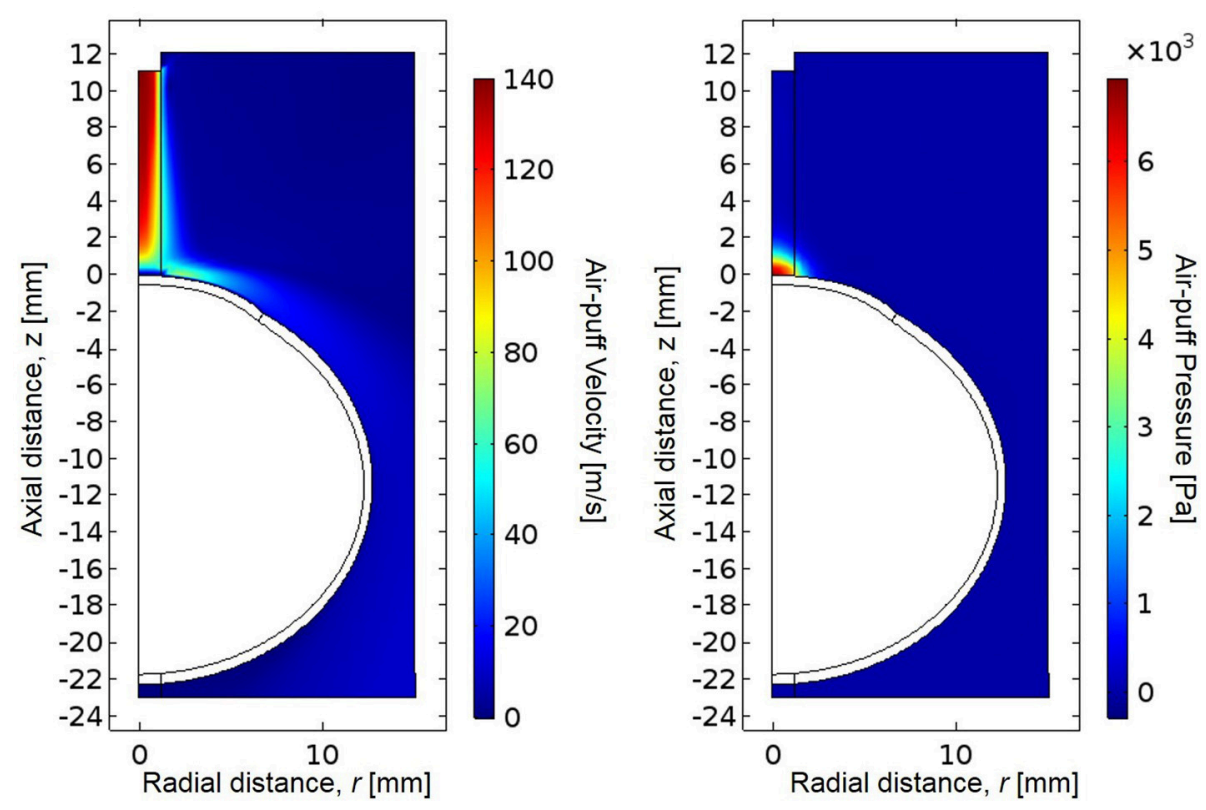

FIGURE 5 | Simulated CorVis ST air-puff velocity profile (left) and pressure profile (right), which serves as the load on the anterior boundary of the globe. The simulation profiles agree within $2 \%$ of experimental measurements using hot-wire anemometry from Roberts et al. (2017). 
glutaraldehyde in Dulbecco's phosphate-buffered saline (DPBS) (Sigma-Aldrich, St. Louis, Missouri, USA) just below the level of the limbus, leaving a visible gap such that the cornea remained untreated. Glutaraldehyde visibly stains ocular tissues yellow after a short period of time. The ocular tissues were visually inspected to ensure that the cornea remained unaffected by the glutaraldehyde treatment.

The CorVis ST was used to load the eye and quantify the resulting corneal deformation response (Figure 4). Each eye was secured in a custom whole-globe mount using shallow sutures in the sclera. A 22-gauge needle attached to a saline column was inserted into the anterior chamber of the eye to set and maintain IOP. The column was set to specified heights to generate IOPs corresponding to 10, 20, 30, and $40 \mathrm{mmHg}$. At least 3 examinations were performed at each pressure step, with DPBS dripped onto the cornea between examinations to maintain hydration. All data were acquired within $48 \mathrm{~h}$ post-mortem.

\section{RESULTS}

The CorVis ST air-puff velocity profile and corresponding pressure profile were simulated in COMSOL 5.2a (Figure 5).

TABLE 4 | Spatial measurements of air flow velocity along axis of symmetry at peak pressure.

\begin{tabular}{lccc}
\hline $\begin{array}{l}\text { Axial distance, } \mathbf{z} \\
{[\mathbf{m m}]}\end{array}$ & $\begin{array}{c}\text { Simulation } \\
{[\mathrm{m} / \mathbf{s}]}\end{array}$ & $\begin{array}{c}\text { Hot wire (Roberts } \\
\text { et al., 2017) [m/s] }\end{array}$ & $\begin{array}{c}\text { Error } \\
{[\%]}\end{array}$ \\
\hline 11 & 140.524 & 140.218 & 0.218 \\
9 & 138.394 & 137.725 & 0.486 \\
7 & 136.440 & 137.609 & -0.850 \\
5 & 135.186 & 136.697 & -1.105 \\
3 & 134.616 & 135.337 & -0.533 \\
1 & 134.178 & 133.238 & 0.706
\end{tabular}

The spatial velocity profile agreed within $2 \%$ of experimental measurements by hot wire anemometry (Roberts et al., 2017) along the centerline/axis of symmetry as well as the central corneal region (Tables 3,4 ). The coordinate system for the model has the undeformed corneal apex at the origin, where $r$ indicates the radial distance from the axis of symmetry, and $\mathrm{z}$ indicates the axial distance from the undeformed corneal apex. The air-puff pressure is concentrated at the central corneal region.

The simulated pressure profile was applied as the boundary load on the anterior boundary of the eye. The resulting stress magnitudes were highest at the posterior surface of the corneal apex, which experiences the greatest total deformation and strain (Figure 6). The stresses are also higher at the posterior sclera where the eye was fixed in this simulation. There is an increase in the magnitude of stress near the area of the limbus (interface of cornea and sclera) due to the dissimilar stiffnesses between cornea and sclera in the model. A sensitivity analysis was performed to show model independence from the vitreous body properties (Table 5).

The corneal deformation response produced by the model matched the expected trend (Table 6). For each value of IOP tested, increasing the ratio of scleral to corneal Young's moduli resulted in decreasing maximum apical displacement (Figure 7). Further, increasing the value of IOP while keeping other material properties constant also resulted in decreasing maximum apical displacement, which is consistent with with the results of our

TABLE 5 | Sensitivity analysis of model dependence and vitreous body.

\begin{tabular}{lcc}
\hline $\begin{array}{l}\text { Vitreous shear } \\
\text { modulus [Pa] }\end{array}$ & $\begin{array}{c}\text { Apical } \\
\text { displacement [mm] }\end{array}$ & $\begin{array}{c}\text { Percent change } \\
\text { from 7.5 Pa [\%] }\end{array}$ \\
\hline 0.75000 & -0.81888 & 0.147 \\
7.5000 & -0.81768 & - \\
75.000 & -0.81002 & 0.937
\end{tabular}
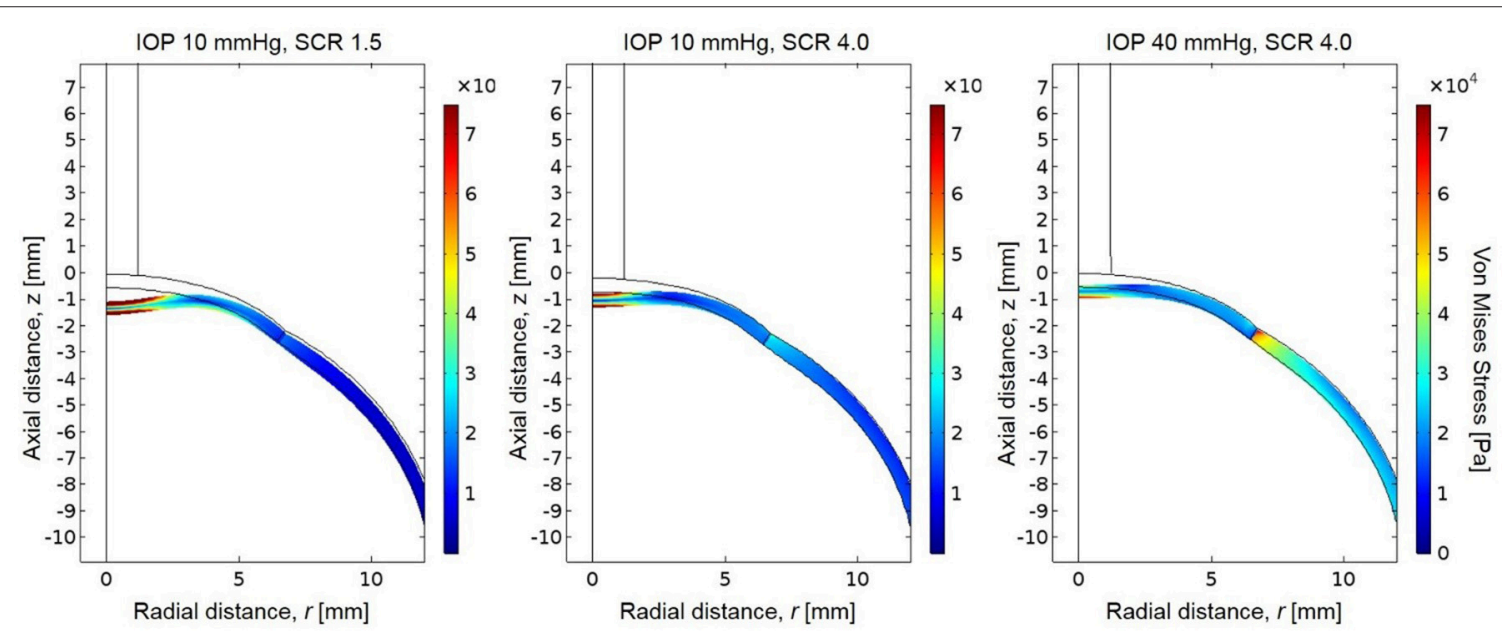

FIGURE 6 | Resultant von Mises stresses and deformation for a globe with scleral-to-corneal ratio of Young's moduli of 1.5 and IOP of 10 mmHg (left), scleral-to-corneal ratio of 1.5 and IOP of $40 \mathrm{mmHg}$ (center), and a globe with scleral-to-corneal ratio of $4.0 \mathrm{and} \mathrm{IOP}$ of $40 \mathrm{mmHg}$ (right). 
TABLE 6 | Summary of simulation results from FE model.

\begin{tabular}{ccc}
\hline IOP [mmHg] & $\begin{array}{c}\text { Scleral-to-corneal } \\
\text { ratio [MPa/MPa] }\end{array}$ & $\begin{array}{c}\text { Max apical } \\
\text { displacement [mm] }\end{array}$ \\
\hline 10 & 1.5 & -1.079 \\
2 & -0.954 \\
3 & -0.818 \\
4 & -0.743 \\
20 & 1.5 & -0.940 \\
& 2 & -0.818 \\
& 3 & -0.689 \\
30 & 4 & -0.569 \\
& 1.5 & -0.848 \\
& 2 & -0.733 \\
& 3 & -0.620 \\
40 & 4 & -0.549 \\
& 1.5 & -0.786 \\
& 2 & -0.682 \\
& 3 & -0.598 \\
4 & -0.492
\end{tabular}

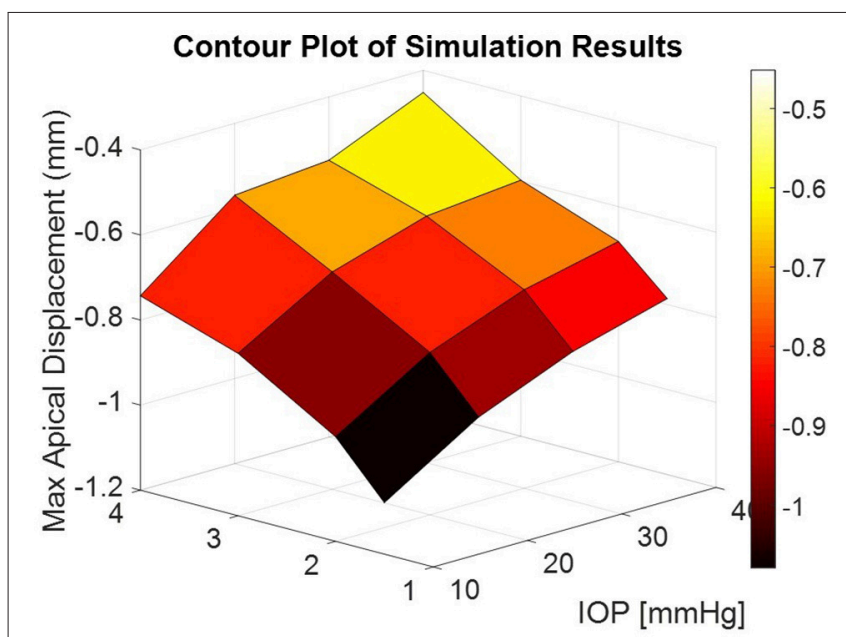

Scleral/Corneal Young's Moduli

FIGURE 7 | Contour plot of maximum apical displacement predicted by the finite-element model as a function of IOP and scleral-to-corneal ratio of Young's moduli. The biomechanical response is non-linear with both increasing $\mathrm{IOP}$ and increasing sclera-to-corneal ratio.

ex vivo experiments (Table 7). Figure 8 shows that the effective stiffness of the cornea increases with higher IOP, resulting in decreased maximum apical displacement in both the FE model and ex vivo results. The simplified Nehookean approximation may contribute to the why the model does not completely bound ex vivo observations.

\section{DISCUSSION}

Scleral stiffness significantly influenced corneal apical displacement in both simulations and ex vivo experiments.
TABLE 7 | Summary of ex vivo results from human donor eyes (Nguyen et al., 2018).

\begin{tabular}{llc}
\hline IOP $[\mathrm{mmHg}]$ & Group & Max apical displacement $[\mathrm{mm}]$ \\
\cline { 3 - 3 } & & Mean \pm Std. Dev. \\
\hline 10 & Control & $-1.494 \pm 0.191$ \\
& Treated & $-1.216 \pm 0.148$ \\
20 & Control & $-0.992 \pm 0.114$ \\
30 & Treated & $-0.891 \pm 0.088$ \\
& Control & $-0.706 \pm 0.068$ \\
40 & Treated & $-0.663 \pm 0.071$ \\
& Control & $-0.520 \pm 0.051$ \\
& Treated & $-0.487 \pm 0.051$
\end{tabular}

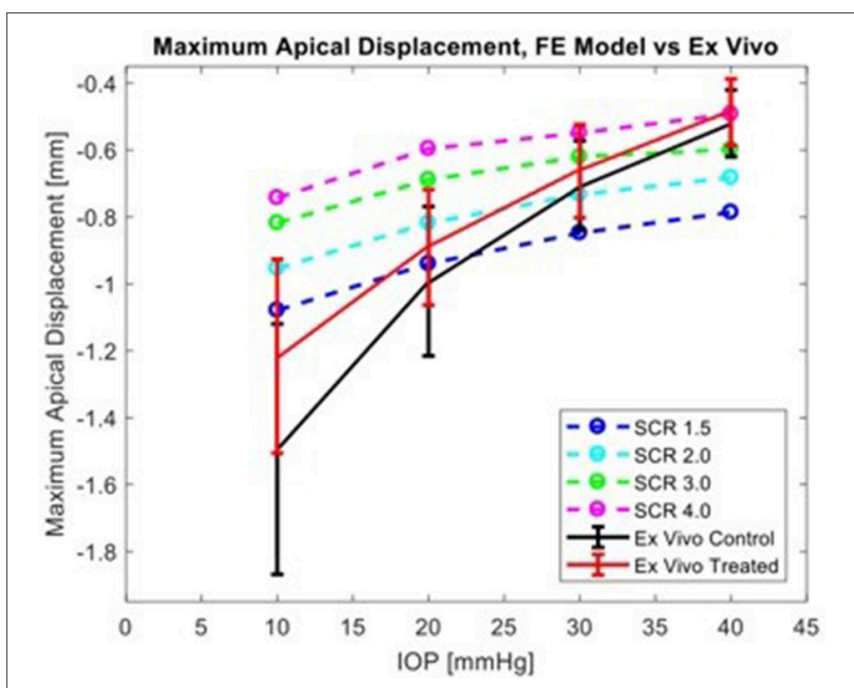

FIGURE 8 | Comparison of simulation results from FE model for several levels of scleral-to-corneal ratio of Youngs' Modulus (SCRs) against ex vivo data. Error bars represent 95\% confidence intervals.

Thus, with increasingly stiff sclera, the greater the limitation on corneal deformation to an air-puff at physiologic levels of IOP. The ex vivo results are consistent with the trends observed by Metzler et al., where the untreated globe undergoes stress stiffening at higher IOP and behaves similarly to the treated globe. This has important clinical implications, where the corneal biomechanical response is often attributed solely to the corneal properties. The maximum apical displacement of the cornea decreased non-linearly with increasing IOP, which is consistent with the principles of tonometry and literature reports (Metzler et al., 2014). Because traditional methods for biomechanical evaluation are limited to ex vivo studies, finite-element (FE) models have been explored as a way to evaluate in vivo properties and response (Elsheikh, 2010; Girard et al., 2015; Pandolfi and Boschetti, 2015; Ariza-Gracia et al., 2016). In published models that simulate corneal deformation by an air-puff, some do not include the sclera (Elsheikh et al., 2009, 2013; Kling et al., 2014; Lago et al., 2014; Pandolfi and Boschetti, 2015; Sinha Roy et al., 2015; Bekesi et al., 2016; Simonini and Pandolfi, 2016). The 
inseparable impact of the sclera to limiting corneal deformation response requires a whole-eye model. Further, the simulated load must be accurate because the biomechanical response is load-dependent.

This FE model, while simple, achieved qualitative agreement with ex vivo experiments. Several aspects of this result give insights which can be used to inform other models. Several models of air puff-induced deformation are comprised of only the cornea, with a variety of scleral boundary conditions applied at the limbus (Elsheikh et al., 2009, 2013; Kling et al., 2014; Lago et al., 2014; Pandolfi and Boschetti, 2015; Sinha Roy et al., 2015; Bekesi et al., 2016; Simonini and Pandolfi, 2016). Our findings indicate that this simplifying representation of the scleral influence on the limbus is inaccurate and introduces systematic errors in these models. Non-contact tonometry devices estimate IOP by observing the response of the cornea; our findings demonstrate that clinical interpretations which neglect scleral contributions to the corneal response to an air-puff may lead to incorrect conclusions. This is particularly important in conditions where the sclera is altered, such as prostaglandin treatment in glaucoma (Toris et al., 2008; Alm and Nilsson, 2009) - which increases scleral permeability and uveoscleral outflow-and progressive myopia (McBrien and Gentle, 2003; Harper and Summers, 2015) - in which the sclera experiences significant collagen remodeling. Reductions in IOP have been reported in some procedures that alter the sclera to treat presbyopia; this is likely artifact from the altered sclera, and there is little or no actual change in IOP (Fukasaku and Marron, 2001; Hipsley et al., 2017).

The present model accurately replicated the velocity and pressure profiles of the CorVis ST air-puff. Similar to the work done by Elsheikh et al. the model also estimated the stressfree state of the eye and generated the residually-stressed state before deformation to more accurately describe the stresses in the cornea and sclera and the resultant biomechanical response (Elsheikh et al., 2013). Finally, a significant advantage of this model is that the simulation results are qualitatively validated with data from ex vivo human donor eyes, in an experimental setup that was replicated closely in the FE model.

One limitation of this model is that it does not account for the dynamic time-dependent response of the cornea to the airpuff. The cornea is a soft biological tissue and therefore exhibits viscoelasticity in its biomechanical response. To account for the non-linear behavior of the tissue, we chose to consider the ocular tissues as hyperelastic, resulting in a non-linear response to increasing IOP. The use of an incompressible neo-Hookean

\section{REFERENCES}

Alm, A., and Nilsson, S. F. (2009). Uveoscleral outflow-A review. Exp. Eye Res. 88, 760-768. doi: 10.1016/j.exer.2008.12.012

Ambrósio R., Jr, Ramos, I., Luz, A., Faria-Correia, F., Steinmueller, A., Krug, M., et al. (2013). Dynamic Ultra-High-Speed Scheimpflug imaging for assessing corneal biomechanical properties. Rev. Bras. Oftalmol. 72, 99-102. doi: 10.1590/S0034-72802013000200005 constitutive model is a simplification: the cornea and sclera are complex structures with significant anisotropy (Nguyen, 2016). In this case, the model effectively assumed that the secant modulus is constant regardless of the magnitude of deformation. It is therefore not surprising that the experimental response shows a much larger dependence on SCR and behaves in a strainstiffening manner. Inclusion of more accurate material models, such as Mooney-Rivlin, will significantly improve the model's ability to make quantitative predictions. Another limitation of this model it does not capture the dynamic nature of the air-puff test. Despite this simplification, the steady-state approximation produced results which qualitatively matched those from ex vivo experiments on human donor tissues. Future iterations of the model will include the dynamic response of the cornea to an air-puff.

The finite-element model presented in this paper demonstrates that scleral material properties have an important impact on the biomechanical deformation response of the cornea in air-puff induced deformation. Namely, the stiffer the sclera, the greater the limitation on corneal deformation. This may have important clinical implications. Often in the clinic, the observed biomechanical deformation response of the cornea is attributed solely to the material properties of the cornea, as well as IOP. However, it is clear from these simulations and experiments that the deformation response of the same cornea varies significantly with varying scleral properties. This suggests that when looking at air-puff induced deformation, that the observed biomechanical response is a result of the combination of both corneal and scleral material properties, in addition to IOP.

\section{DATA AVAILABILITY STATEMENT}

The datasets analyzed for the current study are available from the corresponding author on reasonable request.

\section{AUTHOR CONTRIBUTIONS}

$\mathrm{BN}, \mathrm{MR}$, and CR contributed to this study. BN and CR conceived the study design and all authors contributed to the development of the model. All authors contributed to manuscript revision, read and approved the submitted version.

\section{FUNDING}

This work was supported by NIH/NEI R01 EYE027399. 
Coudrillier, B., Tian, J., Alexander, S., Myers, K. M., Quigley, H. A., and Nguyen, T. D. (2012). Biomechanics of the human posterior sclera: age- and glaucomarelated changes measured using inflation testing. Invest. Opthalmol. Vis. Sci. 53, 1714-1728. doi: 10.1167/iovs.11-8009

Elsheikh, A. (2010). Finite-element modeling of corneal biomechanical behavior. J. Ref. Surg. 26, 289-300. doi: 10.3928/1081597X-20090710-01

Elsheikh, A., Geraghty, B., Alhasso, D., Knappett, J., Campanelli, M., and Rama, P. (2009). Regional variation in the biomechanical properties of the human sclera. Exp. Eye Res. 90, 624-633. doi: 10.1016/j.exer.2010.02.010

Elsheikh, A., and Wang, D. (2007). Numerical modelling of corneal biomechanical behaviour. Comp. Methods Biomech. Biomed. Eng. 10, 85-95. doi: 10.1080/10255840600976013

Elsheikh, A., Whitford, C., Hamarashid, R., Kassem, W., Joda, A., and Buchler, P. (2013). Stress free configuration of the human eye. Med. Eng. Phys. 35, 211-216. doi: 10.1016/j.medengphy.2012.09.006

Fukasaku, H., and Marron, J. A. (2001). Anterior ciliary sclerotomy with silicone expansion plug implantation: effect on presbyopia and intraocular pressure. Int. Ophthalmol. Clin. 41, 133-141. doi: 10.1097/00004397-200104000-00013

Geraghty, B., Jones, S. W., Rama, P., Akhtar, R., and Elsheikh, A. (2012). Agerelated variations in the biomechanical properties of human sclera. JMBBM 16, 181-191. doi: 10.1016/j.jmbbm.2012.10.011

Girard, M. J. A., Dupps, W. J., Baskaran, M., Scarcelli, G., Yun, S. H., Quigley, H. A., et al. (2015). Translating ocular biomechanics into clinical practice: current state and future prospects. Curr. Eye Res. 40, 1-18. doi: 10.3109/02713683.2014.914543

Hamilton, K. E., and Pye, D. C. (2008). Young's modulus in normal conreas and the effect on applanation tonometry. Optom. Vis. Sci. 6, 445-450. doi: 10.1097/opx.0b013e3181783a70

Harper, A. R., and Summers, J. A. (2015). The dynamic sclera: extracellular matrix remodeling in normal ocular growth and myopia development. Exp. Eye Res. 133, 100-111. doi: 10.1016/j.exer.2014.07.015

Heys, J. J., Barocas, V. H., and Taravella, M. J. (2001). Modeling passive mechanical interaction between aqueous humor and iris. J. Biomed. Eng. 123, 540-547. doi: $10.1115 / 1.1411972$

Hipsley, A., Ma, D. H., Sun, C. C., Jackson, M. A., Goldberg, D., and Hall, B. (2017). Visual outcomes 24 months after LaserACE. Eye Vis. 4:15. doi: 10.1186/s40662-017-0081-y

Hon, Y., and Lam, A. K. C. (2013). Corneal deformation measurement using scheimpflug noncontact tonometry. Optom. Vis. Sci. 90, e1-e8. doi: 10.1097/OPX.0b013e318279eb87

Kling, S., Bekesi, N., Dorronsoro, C., Pascual, D., and Marcos, S. (2014). Corneal viscoelastic properties from finite-element analysis of in vivo air-puff deformation. PLOS ONE 9:e104904. doi: 10.1371/journal.pone.01 04904

Lago, M. A., Ruperez, M. J., Martinez-Martinez, F., Monserrat, C., Larra, E., Guell, J. L., et al. (2014). A new methodology for the in vivo estimation of the elastic constants that characterize the patient-specific biomechanical behavior of the human cornea. J. Biomech. 48, 38-43. doi: 10.1016/j.jbiomech.2014.11.009

Liu, J., and Roberts, C. J. (2005). Influence of corneal biomechanical properties on intraocular pressure measurement: quantitative analysis. JCRS 31, 146-155. doi: $10.1016 /$ j.jcrs.2004.09.031

Luce, D. A. (2005). Determining in vivo biomechanical properties of the cornea with an ocular response analyzer. J. Cataract. Refract. Surg. 31, 156-162. doi: $10.1016 /$ j.jcrs.2004.10.044

McBrien, N. A., and Gentle, A. (2003). Role of the sclera in the development and pathological complications of myopia. Prog. Retinal Eye Res. 22, 307-338. doi: 10.1016/S1350-9462(02)00063-0

McKee, C. T., Last, J. A., Russel, P., and Murphy, C. J. (2011). Indendation versus tensile measurements of Young's modulus for soft biological tissues. Tissue Eng. Part B Rev. 17, 166-164. doi: 10.1089/ten.teb.2010.0520

Metzler, K. M., Mahmoud, A. M., Liu, J., and Roberts, C. J. (2014). Deformation response of paired donor corneas to an air-puff: intact whole globe versus mounted corneoscleral rim. J. Cataract. Refract. Surg. 40, 888-896. doi: 10.1016/j.jcrs.2014.02.032

Ng, E. Y. K., and Ooi, E. H. (2006). FEM simulation of the eye structure with bioheat analysis. Comp. Methods Program. Biomed. Eng. 82, 268-276. doi: 10.1016/j.cmpb.2006.04.001

Nguyen, B. A., Roberts, C. J., and Reilly, M. A. (2018). Preliminary study on biomechanical contribution of the sclera to dynamic corneal response in air-puff induced deformation. Invest. Ophthalmol. Vis. Sci. 59, 1410.

Nguyen, T. D. (2016). "Biomechanics of the cornea and sclera," in Structure-Based Mechanics of Tissues and Organs, eds G. Kassab and M. Sacks (Boston, MA: Springer), 285-315.

Norman, R. E., Flanagan, J. G., Rausch, S. M., Sigal, I. A., Tertinegg, I., Eilaghi, A., et al. (2010). Dimensions of the human sclera: thickness measurement and regional changes with axial length. Exp. Eye Res. 90, 277-284. doi: 10.1016/j.exer.2009.11.001

Pandolfi, A., and Boschetti, F. (2015). The influence of the geometry of the porcine cornea on the biomechanical response of inflation tests. Comp. Methods Biomech. Biomed. Eng. 18, 64-77. doi: 10.1080/10255842.2013. 778983

Roberts, C. J. (2014). Concepts and misconceptions in corneal biomechanics. JCRS 40, 862-869. doi: 10.1016/j.jcrs.2014.04.019

Roberts, C. J. (2016). Importance of accurately assessing biomechanics of the cornea. Curr. Opin. Ophthalmol. 27, 285-291. doi: 10.1097/ICU.0000000000000282

Roberts, C. J., Mahmoud, A. M., Bons, J. P., Hossain, A., Elsheikh, A., Vinciguerra, R., et al. (2017). Introduction of two novel stiffness parameters and interpretation of air-puff induced biomechanical deformation parameters with a dynamic scheimpflug analyzer. J. Refract. Surg. 33, 266-273. doi: 10.3928/1081597X-20161221-03

Ruberti, J. W., Roy, A. S., and Roberts, C. J. (2011). Corneal biomechanics and biomaterials. Annu. Rev. Biomed. Eng. 13, 269-295. doi: 10.1146/annurev-bioeng-070909-105243

Simonini, I., and Pandolfi, A. (2016). The influence of intraocular pressure and air jet pressure on corneal contactless tonometry tests. JMBBM 2016, 75-89. doi: 10.1016/j.jmbbm.2015.07.030

Sinha Roy, A., Kurian, M., Matalia, H., and Shetty, R. (2015). Air-puff associated quanitifaction of non-linear biomechanical properties of the human cornea in vivo. J. Mech. Behav. Biomed. Mater. 48, 173-182. doi: 10.1016/j.jmbbm.2015.04.010

Tang, J., and Liu, J. (2012). Ultrasonic measurement of scleral cross-sectional strains during elevations of intraocular pressure: method validation and initial results in posterior porcine sclera. J. Biomed. Eng. 134:091007. doi: 10.1115/1.4007365

Toris, C. B., B'Ann, T. G., and Kaufman, P. L. (2008). Update on the mechanism of action of topical prostaglandins for intraocular pressure reduction. Survey Ophthalmol. 53, S107-S120. doi: 10.1016/j.survophthal.2008.08.010

Vojniković, B., Gabrić, N., Dekaris, I., and Jurić, B. (2013). Curvature analyses of the corneal front and back surface. Coll. Antropol. 37, 93-96.

Conflict of Interest Statement: CR is a consultant for Oculus Optikgeräte GmbH.

The remaining authors declare that the research was conducted in the absence of any commercial or financial relationships that could be construed as a potential conflict of interest.

Copyright (c) 2019 Nguyen, Roberts and Reilly. This is an open-access article distributed under the terms of the Creative Commons Attribution License (CC BY). The use, distribution or reproduction in other forums is permitted, provided the original author(s) and the copyright owner(s) are credited and that the original publication in this journal is cited, in accordance with accepted academic practice. No use, distribution or reproduction is permitted which does not comply with these terms. 